\title{
A variant of long QT syndrome manifested as fetal tachycardia and associated with ventricular septal defect
}

\author{
M-H Wu, F-C Hsieh, J-K Wang, M-L Kau
}

\begin{abstract}
Two patients with a novel variant of long QT syndrome are described. The clinical course was characterised by an in utero onset of ventricular tachycardia and atrioventricular block (at 26 and 30 weeks' gestational age, respectively), and an association with a ventricular septal defect. Studies of both patients' families identified relatives with prolonged QT interval, syncope, or sudden death. One patient died of intractable ventricular tachycardia at 4 days old. The other received $\beta$ blocker treatment and a pacemaker. She died suddenly at the age of 10 months. The unique association with ventricular septal defect and the malignant clinical course warrants further molecular diagnosis of this novel variant of long QT syndrome.

(Heart 1999;82:386-388)
\end{abstract}

Keywords: long QT syndrome; ventricular septal defect; fetal tachycardia

Congenital long QT syndrome is an inherited disorder with prolonged ventricular repolarisation and a propensity to sudden cardiac death caused by a polymorphic ventricular tachycar-

ccepted for publication 17 March 1999
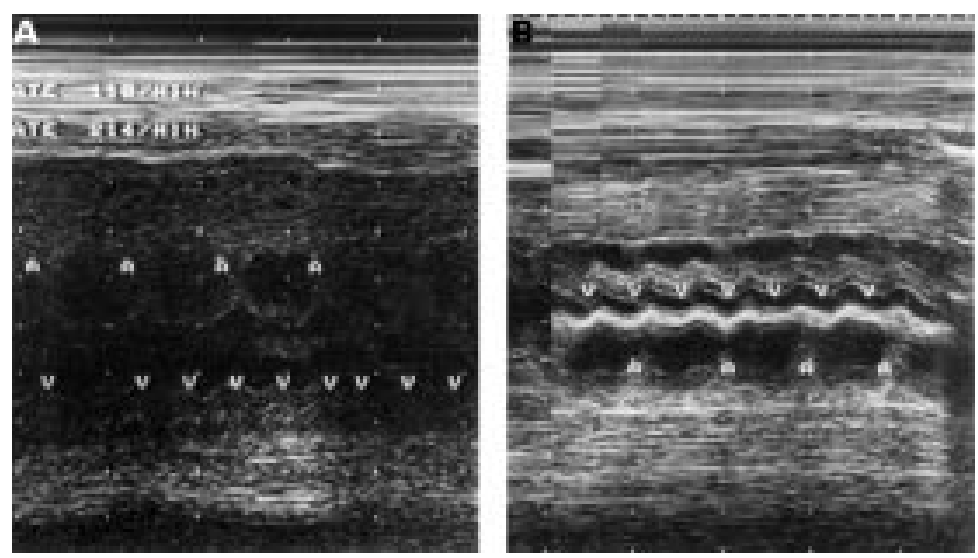

Figure 1 (A) $M$ mode fetal echocardiogram positioned to record simultaneously the left ventricle (V) and left atrium (A). Ventricular tachycardia was diagnosed because of the rapid ventricular rate with dissociated ventriculo-atrial conduction. (B) $M$ mode fetal echocardiogram of case 2. The aortic root and the left atrium were simultaneously recorded. The aortic valve opening represented the ventricular activity $(\mathrm{V})$ and the left atrial motion represented the atrial activity (A). Ventricular tachycardia was diagnosed based on the rapid ventricular rate with dissociated ventriculo-atrial conduction. dia "torsade de pointes". ${ }^{1}$ It is usually not associated with congenital heart disease. ${ }^{12}$ Patients with long QT syndrome may develop atrioventricular (AV) block or be noted early in the neonatal stage. ${ }^{34}$ Only very rarely may ventricular tachycardia and intermittent AV block occur during fetal life. ${ }^{5}$ After the description of a single case with fetal ventricular tachycardia, we have identified another patient with similar clinical presentation. ${ }^{5}$ Both had intermittent ventricular tachycardia and AV block during fetal life, and both died during infancy. The family history revealed family members with sudden death, syncope, or prolonged QT interval. In both patients, a perimembranous ventricular septal defect was also found. Therefore, a novel variant of congenital long QT syndrome is proposed based on the clinical characteristics of early (in utero) onset and malignant nature of the arrhythmias, and the association with ventricular septal defect.

\section{Case 1}

A full term baby girl (weight $2726 \mathrm{~g}$ ) was born to a 25 year old mother. At 26 weeks' gestation, she was found to have intermittent bradycardia. Fetal echocardiogram showed sinus bradycardia, intermittent AV block (2:1 to 6:5) and tachycardia with ventriculo-atrial dissociation (fig 1A). Maternal anti-dsDNA was $2.42 \mathrm{iu} / \mathrm{ml}$. Anti ENA (including SM, RNP, Ro, La and Scl-70) were all negative. The $\mathrm{C}_{3}$ and $\mathrm{C}_{4}$ were within normal range. An ECG taken soon after birth showed sinus rhythm with prolonged QT interval $\left(\mathrm{QT}_{\mathrm{c}}=0.65\right.$ seconds, by Bazett formula) (fig 2A). The echocardiogram revealed a perimembranous ventricular septal defect with aneurysmal transformation. The auditory brain stem evoked potential was normal. The patient's family history revealed her uncle had a sudden unexplained death and her mother had syncope (fig 3A).

The patient developed second degree AV block with alternate left and right bundle branch block at 3 days old. She subsequently received propranolol $(1.5 \mathrm{mg} / \mathrm{kg} /$ day $)$ and an epicardial pacemaker. The night before the pacemaker implantation, ventricular tachycardia (torsade de pointes) developed, which was converted by DC conversion and lidocaine. The patient progressed well and pacemaker 
A
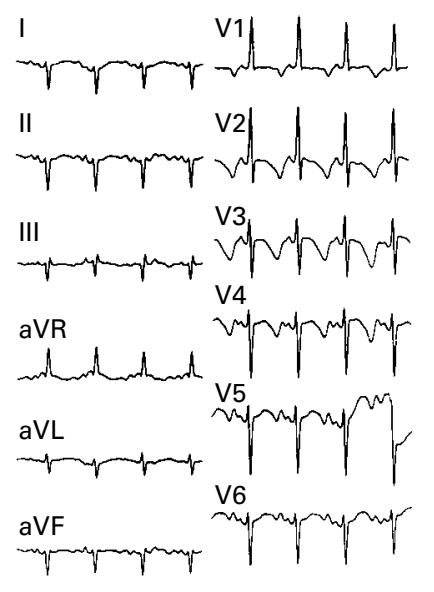

\section{B}
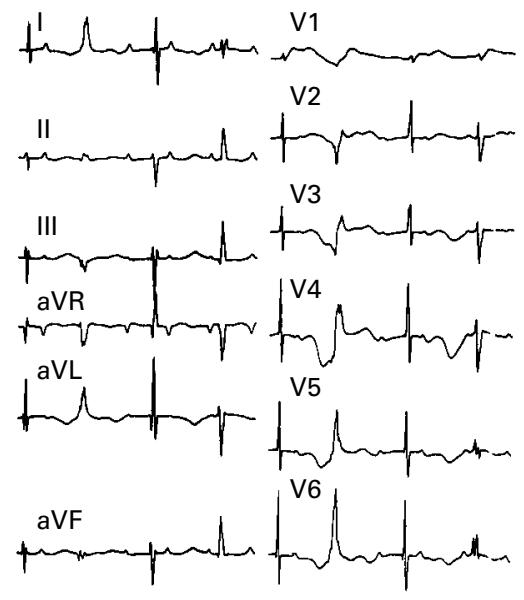

II

Figure 2 (A) Standard ECG of case 1 taken soon after birth. Paper speed $25 \mathrm{~mm} / \mathrm{s}$; $1 \mathrm{mV}=10 \mathrm{~mm}$. (B) Standard ECG of case 2 recorded at 2 days old. Paper speed $25 \mathrm{~mm} / \mathrm{s}, 1 \mathrm{mV}=10 \mathrm{~mm}$. The temporary pacemaker was turned off. (C) Rhythm strip (lead 2) of case 2 recorded at 2 days old. Paper speed $25 \mathrm{~mm} / \mathrm{s} ; 1 \mathrm{mV}=10 \mathrm{~mm}$.

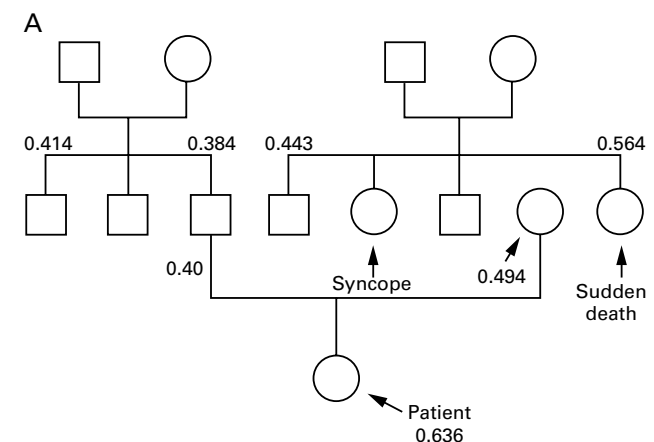

B

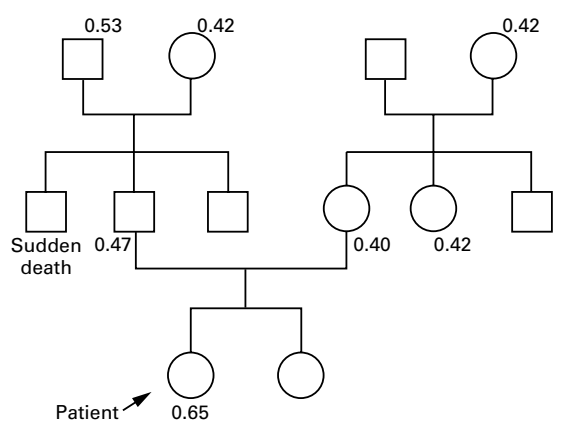

Figure 3 Family history of: $(A)$ case 1; and (B) case 2.

function was normal at monthly visits. However, at 10 months old she was shocked by a loud noise from a truck. On cardiopulmonary resuscitation, recurrent ventricular tachycardia was noted and she died of multiorgan failure the next day.

Case 2

A full term baby girl (weight $3276 \mathrm{~g}$ ) was noted to have bradycardia at 30 weeks' gestational age. A fetal echocardiogram revealed intermittent tachycardia with ventriculo-atrial dissociation (fig 1B) and AV block. In addition, a peri- membranous ventricular septal defect was found. Maternal lupus studies were all negative. Soon after birth she was found to have high degree AV block (ventricular rate 27-90 beats/min) and intermittent ventricular tachycardia. The QT interval was 0.64 seconds, and 0.86 seconds after correction (fig $2 \mathrm{~B}, \mathrm{C}$ ). She received an emergency temporary pacemaker. Echocardiography confirmed the defect. However, the patient died of intractable ventricular tachycardia at 4 days old. Family study revealed her uncle died of sudden death, and her father and grandfather had prolonged $\mathrm{QT}_{\mathrm{c}}$ (fig 3B).

\section{Discussion}

We describe a unique form of congenital long QT syndrome which is characterised by an early intrauterine onset of the dysrhythmia and an association with congenital heart disease, a perimembranous ventricular septal defect. The malignant clinical course warrants special consideration.

Patients with long QT syndrome are prone to develop life threatening ventricular tachycardia, especially torsade de pointes ventricular tachycardia. ${ }^{1}$ The symptoms of long QT syndrome may surface at any age as episodic dizziness, palpitations, syncope, and even cardiac death. It may also be involved in some cases of sudden infant death. ${ }^{7}$ Nonetheless, the occurrence of ventricular tachycardia during fetal life has rarely been described. ${ }^{56}$ Hofbeck and colleagues retrospectively analysed the fetal echocardiograms of nine neonates with long QT syndrome and found two of them with in utero ventricular tachycardia. ${ }^{6}$ Both died at 1 day old despite treatment with propranolol and transvenous pacing. Intermittent AV block was documented in both. Our patients similarly developed ventricular tachycardia and intermittent AV block during the fetal life. Both needed early pacemaker treatment and died of intractable ventricular tachycardia during infancy. They are distinct in that they had coexisting congenital heart disease in the form of a perimembranous ventricular septal defect.

The prevalence of congenital heart disease is 4-7 per 1000 live births, with ventricular septal defect ranging from $16-43 \% .{ }^{8}$ However, none of the previous reports addressed the association between the defect and long QT syndrome. The incidence of symptomatic long QT syndrome during the perinatal stage was even more rare. ${ }^{2}{ }^{6}$ Only one report describes a patient with long QT syndrome and congenital heart disease (ventricular septal defect, moderate degree of right ventricular hypoplasia, and spongy myocardium) who had syncope at the age of 6 months and was resuscitated successfully. ${ }^{6}$ Both our cases had a perimembranous ventricular septal defect. Although the coexistence may be incidental, an association between ventricular septal defect and a particular variant of long QT syndrome should be taken into consideration.

Recent studies have focused on the molecular genetics of long QT syndrome. ${ }^{9}$ Three separate genetic loci for the long QT syndrome, including mutations in the sodium or 
potassium channels, have been identified. Those genetic abnormalities may be associated with different phenotypic $\mathrm{T}$ wave patterns on ECG. ${ }^{10}$ The $\mathrm{T}$ wave patterns present in our patients share some similarities with those found in patients with abnormal gene loci on chromosome 3 or 7 . However, none of these genetically confirmed patients had fetal onset dysrhythmia or congenital heart disease.

In conclusion, two patients with long QT syndrome developed ventricular tachycardia and intermittent AV block early in their fetal life. Both had perimembranous ventricular septal defect and early mortality caused by intractable ventricular tachycardia. The unique association with a ventricular septal defect and the malignant clinical course (in utero onset and early mortality) warrant further molecular diagnosis of this novel variant of long QT syndrome. Aggressive treatments are suggested for pa-tients with such a variant of long QT syndrome.
1 Schwartz PJ. The long QT syndrome. Curr Probl Cardiology 1997;22:300-47.

2 Mache CJ, Beitzke A, Haidvogl M Jr, et al. Perinatal manifestations of idiopathic long QT syndrome. Pediatr Cardiol 1996;17:118-21.

3 Villain E, Levy M, Kachaner J, et al. Prolonged QT interval in neonates: benign, transient, or prolonged risk of sudden death. Am Heart f 1992;124:194-7.

4 Garson A, Dick M, Fournier A, et al. The long QT syndrome in children: an international study of 287 patients. Circulation 1993;87:1866-72.

5 Lin MT, Wu MH, Hsieh FJ, et al. Long QT syndrome manifested as fetal ventricular tachycardia and intermittent AV block. Am 7 Perinatol 1998;15:145-7.

6 Hofbeck M, Herbert U, Ernst B, et al. Prenatal findings in patients with prolonged QT interval in the neonatal period. Heart 1997;77:198-204.

7 Schwartz PJ, Stramba-Badiale M, Segantini A, et al. Prolongation of the QT interval and the sudden infant death syndrome. N Engl f Med 1998;338:1709-14.

8 Rosenthal G. Prevalence of congenital heart disease. In: Garson A Jr, Bricker JT, Fisher DJ, et al, eds. The science and practice of pediatric cardiology. Baltimore: Williams \& Wilkins, 1998:1083-105.

9 Priori SG, Napolitano C, Paganini V, et al. Molecular biology of the long QT syndrome: impact on management. PACE 1997;20:2052-7.

10 Moss AJ, Zareba W, Benhorin J, et al. ECG T-wave patterns in genetically distinct forms of the hereditary long QT syndrome. Circulation 1995;92:2929-34. 